\title{
Improving Surface Current Resolution Using Direction Finding Algorithms for Multiantenna High-Frequency Radars
}

\author{
ANTHONY KIRINCICH \\ Woods Hole Oceanographic Institution, Woods Hole, Massachusetts \\ BRIAN EMERY AND LIBE WASHBURN \\ University of California, Santa Barbara, Santa Barbara, California \\ PIERRE FlAMENT \\ University of Hawai' $i$ at Mānoa, Honolulu, Hawaii
}

(Manuscript received 18 February 2019, in final form 1 August 2019)

\begin{abstract}
While land-based high-frequency (HF) radars are the only instruments capable of resolving both the temporal and spatial variability of surface currents in the coastal ocean, recent high-resolution views suggest that the coastal ocean is more complex than presently deployed radar systems are able to reveal. This work uses a hybrid system, having elements of both phased arrays and direction finding radars, to improve the azimuthal resolution of HF radars. Data from two radars deployed along the U.S. East Coast and configured as 8-antenna grid arrays were used to evaluate potential direction finding and signal, or emitter, detection methods. Direction finding methods such as maximum likelihood estimation generally performed better than the well-known multiple signal classification (MUSIC) method given identical emitter detection methods. However, accurately estimating the number of emitters present in HF radar observations is a challenge. As MUSIC's direction-of-arrival (DOA) function permits simple empirical tests that dramatically aid the detection process, MUSIC was found to be the superior method in this study. The 8-antenna arrays were able to provide more accurate estimates of MUSIC's noise subspace than typical 3-antenna systems, eliminating the need for a series of empirical parameters to control MUSIC's performance. Code developed for this research has been made available in an online repository.
\end{abstract}

\section{Introduction}

Land-based high-frequency (HF) radar systems (Barrick 1972) have proven to be highly effective at measuring coastal ocean surface currents on an operational basis (Harlan et al. 2010). In the United States and other countries, national HF radar networks contribute data for operational use (e.g., search and rescue, spill response) as well as for use in numerical modeling and forecasting of the coastal ocean. Within the research community, surface current data from HF radars have aided studies of the dynamics of coastal circulation and exchange [see Paduan and Washburn (2013) for a review]. However, HF radar-based estimates of surface currents are limited to fairly broad spatial scales

Corresponding author: Anthony Kirincich, akirincich@whoi.edu by the inherent spatial resolution of the instrument and by the spatial smoothing applied to reduce errors and/or data gaps. Both recent high-resolution model simulations (Fig. 1) as well as satellite images of highresolution sea surface temperature (SST) or sun glint suggest that the surface of the ocean is more complex than HF radar systems report. Thus, HF radar-based observations miss potentially important scales of the near-surface dynamics as well as the true nature of horizontal stirring and surface dispersion, both of which are critical to research and operational applications.

Commercially available HF radar systems generally fall into one of two technologies, beamforming (BF) or direction finding (DF). The first uses widely spaced arrays of antennas, obtains direction by beamforming, and observes the Doppler shift in the direction of the electronically steered beam (Gurgel et al. 1999). The second 

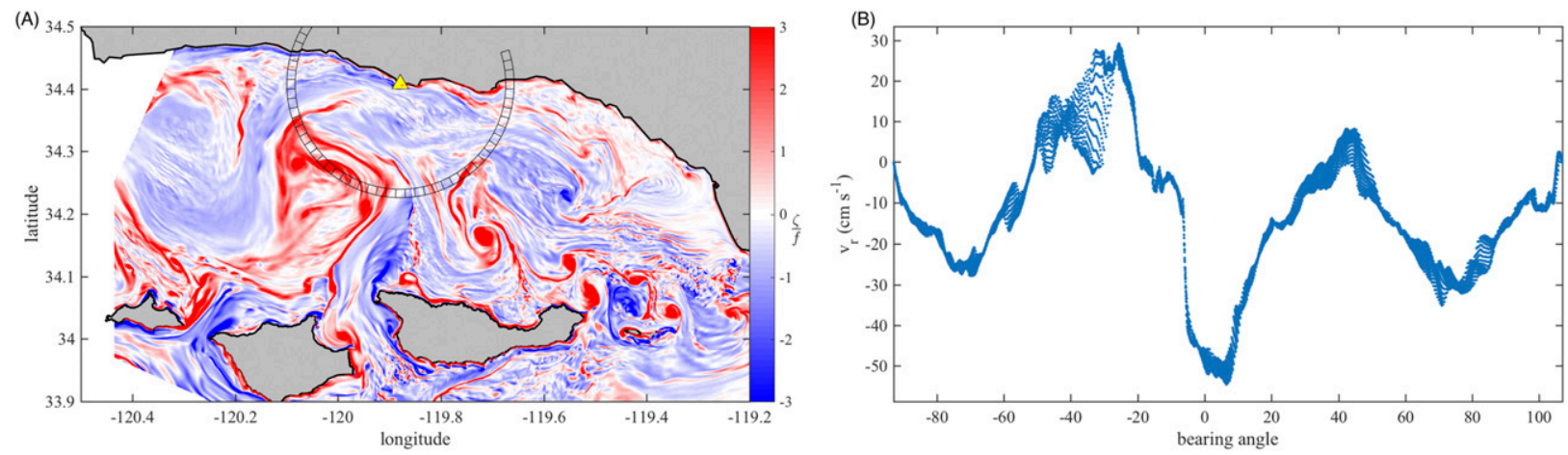

FIG. 1. (a) Snapshot of surface vorticity, as $\zeta / f$, from an ultrahigh (100-m horizontal grid) resolution numerical model of the Southern California Bight [see Romero et al. (2016) for model details]. (b) Radial velocities along the range circle shown in (a), toward (positive) and away (negative) from the radar station in the center of the arc.

uses collocated monopole and loop antennas and applies signal processing algorithms to extract the direction of arrival of signals with distinct Doppler shifts (cf. Lipa et al. 2006; de Paolo et al. 2007). For both technologies, the range resolution $\Delta r \sim c / 2 B$ is governed by the radio bandwidth $B$ allocated ( $c$ is the speed of light). Numerous studies have established the limitations of HF radarbased surface currents using either technology (e.g., Lipa et al. 2006; Wyatt 2005), the total range or the range resolution (Paduan and Washburn 2013), their differences from, and relationships to, in situ observations (e.g., Kohut et al. 2006; Ullman et al. 2006; Ohlmann et al. 2007), and the velocity errors due to instrumental errors (Emery et al. 2004; de Paolo and Terrill 2007; Laws et al. 2010; Kirincich et al. 2012).

However, the role of azimuthal resolution, the spatial resolution along each range circle, defined in bearing, has generally not been addressed. Azimuthal resolution limits the smallest observable horizontal scale over most of a radar's observational extent, and thus is the primary factor limiting high-resolution mapping of surface currents. This can be illustrated by a quick calculation assuming a $5^{\circ}$ azimuthal resolution, which is representative of the typical beamwidth of a 16-antenna phased array radar and also the standard output of the SeaSonde (Lipa et al. 2006). Translating this resolution into kilometers, the azimuthal resolution becomes coarser than the standard International Telecommunication Union (ITU) allocated range resolutions of 1,2 , and $6 \mathrm{~km}$ (for transmit frequencies of 25,13 , and $5 \mathrm{MHz}$, respectively) at offshore ranges of 17,35 , and $70 \mathrm{~km}$, respectively. As radars operating at frequencies of 25,13 , and $5 \mathrm{MHz}$ have nominal ranges of 40,90 , and $180 \mathrm{~km}$, respectively, azimuthal resolution sets the smallest observable scale in over $60 \%-70 \%$ of the nominal range of most radars.

Heterogeneous flows in the coastal ocean add complexity to the raw Doppler spectral data used by radars to derive surface velocities in a number of ways. For the SeaSonde (Lipa et al. 2006), a 3-antenna DF radar, the maximum number of directions that signals, or emitters as defined in the signal processing literature, can arrive from is two per each individual estimate of the radial velocity at a given range. For BF systems configured as a $M$-antenna linear array, such as the Wellen Radar (WERA; Gurgel et al. 1999), the Doppler spectra for each steered beam, or each bearing, is used to produce just one estimate of the radial velocity, integrating all emitters over the beamwidth (a minimum of $\sim 6^{\circ}$ for 16 antennas, depending on the bearing relative to the array). For both types of systems, complex flow structures can yield radial velocities at the same Doppler shift from multiple bearings (e.g., Kirincich and Lentz 2017), and at multiple Doppler shifts within the same azimuthal bin. For SeaSonde DF processing, such complex flows lead to increased errors in identifying first-order Bragg returns (e.g., Kirincich 2017b), and increased multivalued solutions that can overwhelm the 2-emitter limit (Laws et al. 2010; Emery and Washburn 2017; Emery 2018). For $\mathrm{BF}$ processing, these additional emitters can widen the first-order Bragg spectrum and increase uncertainty in the radial velocity estimates (Jeans and Donnelly 1986).

The potential for multivalued solutions in complex flows is illustrated by the model velocity field shown in Fig. 1a (Romero et al. 2016) and considering the radial velocity component along a range circle (i.e., the velocity directed toward or away from the radar), shown as a function of bearing in Fig. 1b. Viewed over a $2 \mathrm{~cm} \mathrm{~s}^{-1}$ span of velocity, equivalent to a typical Doppler radial velocity resolution, identical radial velocities can exist at up to four independent bearings (e.g., $v_{r}=-20 \mathrm{~cm} \mathrm{~s}^{-1}$ ). Furthermore, numerous bearings exist at which the span of velocities within the nominal $5^{\circ}$ azimuthal bin is greater than $10 \mathrm{~cm} \mathrm{~s}^{-1}$ (e.g., $-30^{\circ}$ or $40^{\circ}$ ). Thus, the complexity of flow structures suggested by the model 
exceeds the ability of existing radars to map them accurately, whether based on DF or BF technologies, independent of additional errors such as instrumental noise, phase biases, or antenna pattern inaccuracies.

This work seeks to advance the capabilities of HF radars to measure complex flows. Recent efforts (e.g., Capet et al. 2008; Chavanne et al. 2010a,b; Lentz and Fewings 2012; Rypina et al. 2014; Romero et al. 2016; Kirincich and Lentz 2017) have suggested that spatially complex flows are an important part of the variability present in the coastal ocean and critical to robust estimates of exchange across the coastal zone. An important aspect of increasing the resolution of HF radar systems is improving their ability to measure two or more independent signals that may be closely spaced in bearing or Doppler velocity. This can be accomplished by improving how currents are extracted from radar observations or improving how the radars themselves are configured. The present effort seeks a combination of both, and uses data from two generic 8-channel HF radars configured as rectangular phased arrays to assess a number of promising parameter-based DF methods found in the signal processing literature (Emery 2018) against the multiple signal classification (MUSIC) algorithm employed by the SeaSonde (Lipa et al. 2006). Advances in DF methods for extracting estimates of ocean surface currents are presented first, followed by a description of the experimental setting over the New England shelf. A number of tests are then performed to evaluate the DF methods using comparisons with surface drifters as well as radial velocities estimated from a pair of higher-resolution HF radars. The results of these tests are discussed to interpret their significance, justify the methodology used, and guide future improvements.

\section{Direction-finding methods for HF radar-based surface currents}

\section{a. The covariance matrix}

Direction-finding methods for HF radar-based surface currents are based on estimates of the covariance matrix (C) formed from complex voltages observed by the individual receive antennas. In contrast to target tracking, ocean returns contain signals from all ranges and radial velocities. Two Fourier transforms are performed on time series of the complex voltages, the first to separate signals by range and the second by Doppler frequency, to estimate currents. This is crucial as the ocean surface presents a spatially distributed source of backscattered signals, rather than discrete point sources in Gaussian noise. For a given range $r$ and Doppler frequency $f$, the $M \times M$ covariance matrix $\mathbf{C}=$ $\mathbf{C}(r, f)$, where $M$ is the number of receive antennas, is formed from the self-product and the cross product of the data from all antennas (cf. de Paolo et al. 2007). Critically, even though $\mathbf{C}$ is a time-integrated product over the transformed time series length, each estimate of $\mathbf{C}$ forms only one realization - or snapshot-of the signals observed by the antenna array (e.g., Wang and Gill 2016).

Many of the direction-finding methods described below depend on the eigendecomposition of $\mathbf{C}$ to estimate solutions; $\mathbf{C}$ is a Hermitian matrix with the requirement to be full rank (i.e., to possess $M$ nonzero eigenvalues; Horn and Johnson 1985). This requires more independent estimates $(K)$ of $\mathbf{C}$ than the size of $\mathbf{C}$ (or $K>M$ ), to be averaged into an ensemble before direction finding. Thus, given the nonstationarity of complex coastal currents and the time required to acquire a full-rank ensemble, DF methods for estimating surface currents from $\mathrm{HF}$ radars must perform well with minimal $K$. Additional requirements for oceanographic HF radars include performance in conditions of low signal-to-noise ratios (SNRs), and the ability to extract surface currents over a wide azimuthal area. It should be noted that matrices formed at adjacent ranges and Doppler frequencies (e.g., Fig. 3) are not statistically independent due to the nature of discrete Fourier transforms, the overlapping of the time series segments, and the windowing applied to suppress side lobes, leakage, and spectral noise (Martinez-Pedraja et al. 2013).

\section{b. Direction finding algorithms}

\section{1) MUSIC}

Since the original implementation of MUSIC by Schmidt (1986), its theoretical basis and performance as a direction-of-arrival (DOA) estimator has been thoroughly explored, resulting in a deeper understanding of the limitations of MUSIC, and the parameters that affect its performance (cf. Krim and Viberg 1996; Tuncer and Friedlander 2009). Due to its computational efficiency, and flexibility for the size, shape, and extent of the antenna array, MUSIC is still a leading method (Tuncer and Friedlander 2009). Within the MUSIC algorithm, the DOA function $P_{\text {MUSIC }}$ is computed from the noise eigenvectors $\mathbf{E}_{N}$ of $\mathbf{C}$, for each azimuthal bearing $\theta$ in $\mathbf{A}$ as

$$
P_{\text {MUSIC }}=\frac{1}{\mathbf{A}^{H} \mathbf{E}_{N} \mathbf{E}_{N}^{H} \mathbf{A}},
$$

where the $M \times 1$ vector $\mathbf{A}(\theta)$ describes the complexvalued voltage response of the antenna array to a signal from $\theta$ (cf. Friedlander 2009). In Eq. (1), the $M \times(M-N)$ matrix $\mathbf{E}_{N}$ describes the noise subspace, with its dimension determined by the assumed number of emitters $N$. 
The denominator of Eq. (1) represents the projection of $\mathbf{A}(\theta)$ onto the noise subspace. As $\theta$ approaches the true emitter location, $\mathbf{A}(\theta)$ becomes orthogonal to $\mathbf{E}_{N}$, and the denominator goes to zero. Defining $P_{\text {MUSIC }}$ at all $\theta$ thus produces peaks at the $\theta$ that best approximate the emitter location(s).

\section{2) MLE}

A particular implementation of the maximum likelihood estimation (MLE) method that uses the alternating projection search (MLE-AP; Ziskind and Wax 1988) is applied here due to its computational efficiency over standard MLE methods (cf. Emery 2018). MLE-AP seeks the $\theta_{N}$ that maximize

$$
P_{\mathrm{MLE}}=\operatorname{Tr}\left[\mathbf{A}\left(\mathbf{A}^{H} \mathbf{A}\right)^{-1} \mathbf{A}^{H} \mathbf{C}\right]
$$

where Tr is the matrix trace operator and $\mathbf{A}$ has size $M \times N$. MLE-AP has been demonstrated to have lower errors than MUSIC (Tuncer and Friedlander 2009; Emery 2018) and better angular resolution (Krim and Viberg 1996) particularly for small numbers of array elements (Ziskind and Wax 1988). MLE methods have also been developed for signal sources that are distributed in bearing (MLE-DS; Lee et al. 1997; Read 1999), which might be more representative of the radar backscatter from the ocean surface. Hybrid algorithms that combine MLE and MUSIC by using MUSIC to initialize the search in MLE (Oh and Un 1991; Choi 1999) exist, but are beyond the scope of the present work.

\section{3) WSF}

Weighted subspace fitting (WSF; Stoica and Sharman 1990; Viberg et al. 1991; Krim and Viberg 1996) applies maximum likelihood estimation to the eigenvectors of the covariance matrix, finding the MLE that minimizes the difference between a model of the data, constructed from the antenna array matrix, and the optimally weighted signal eigenvectors (Krim and Viberg 1996; Emery 2018). After computing the eigendecomposition, the WSF method estimates the noise variance from the noise eigenvalues $\lambda_{i}$ of $\mathbf{C}$,

$$
\sigma=\frac{\sum_{i=N-1}^{M} \lambda_{i}}{M-N},
$$

and uses this variance to compute the optimal weights for the matrix of signal eigenvalues $\lambda_{S}$ :

$$
\mathbf{W}=\left(\lambda_{S}-\sigma \mathbf{l}\right)^{2} \lambda_{S}^{-1},
$$

where $\mathbf{I}$ is the $M \times M$ identity matrix. The WSF method then finds the minimum of the function

$$
P_{\mathrm{WSF}}=\operatorname{Re}\left(\log \left\{\operatorname{Tr}\left[\left(\mathbf{I}-\mathbf{A A}^{\dagger}\right) \mathbf{E}_{s} \mathbf{W E}_{s}^{H}\right]\right\}\right) .
$$

Previous simulation-based evaluations found WSF to have similar DOA errors as MLE (Emery 2018), a finding that is tested here using observations.

\section{c. The emitter problem}

Each of the methods above requires an additional, and often external, method of establishing the number of signals, or emitters, present in each (range, Doppler frequency) observation. Determining the true number of emitters is both separate from the actual implementation of the DOA methodology and critical to its accuracy and computational efficiency. Within the signal processing literature, this is known as the detection problem, and both statistical and application-specific empirical, or parametric, methods are used to detect the number of emitters.

\section{1) Statistical Detection Methods}

Two of the most commonly used statistical methodologies are the Akaike information criterion (AIC; Akaike 1974) and the minimum description length (MDL; Wax and Kailath 1985). Both are based on the eigenvalues of the covariance matrix and are evaluated here for their accuracy. For each assumed number of signals, or emitters $N$, the MDL detection score is defined as

$$
\begin{aligned}
D_{\mathrm{MDL}}= & -K(M-N) \log \left[\frac{\left(\prod_{i=N+1}^{M} \lambda_{i}\right)^{1 /(M-N)}}{\frac{\sum_{i=N+1}^{M} \lambda_{i}}{M-N}}\right] \\
& +\frac{1}{2} N(2 M-N+1) \log (K),
\end{aligned}
$$

where the component in brackets is the ratio of the geometric mean and the arithmetic mean of the noise eigenvalues $\lambda_{i}$. The AIC detection score differs from the above by a factor related to the number of snapshots $K$ :

$$
\begin{aligned}
D_{\mathrm{AIC}}= & -K(M-N) \log \left[\frac{\left(\prod_{i=N+1}^{M} \lambda_{i}\right)^{1 /(M-N)}}{\sum_{i=N+1}^{M} \lambda_{i}} \overline{M-N}\right] \\
& +N(2 M-N+1) .
\end{aligned}
$$

Thus, for the small $K$ found in HF radar data processing, the two methodologies are quite similar (e.g., Fig. 2a). In both, the estimated number of emitters is determined 
(A)
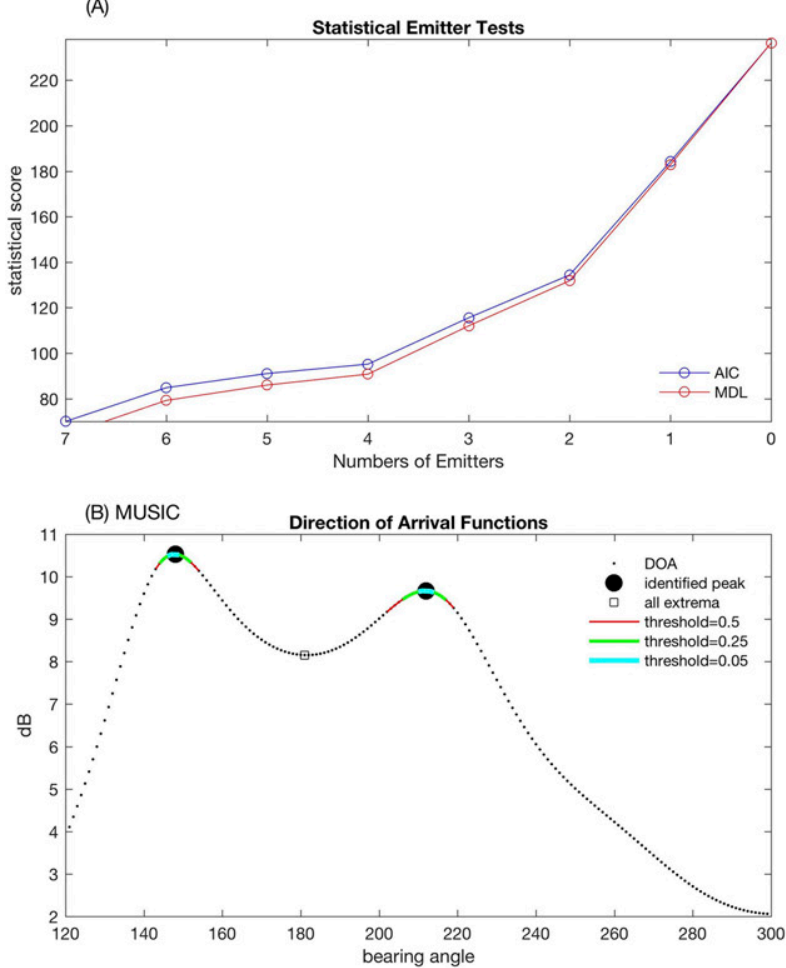

(C) MLE

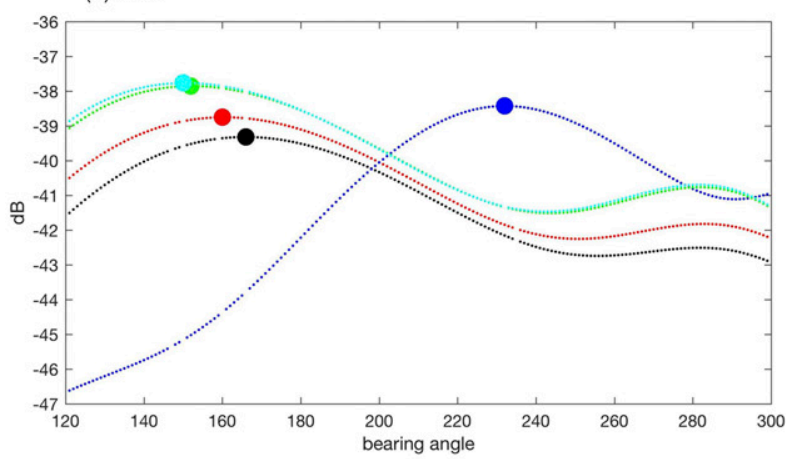

(D) WSF

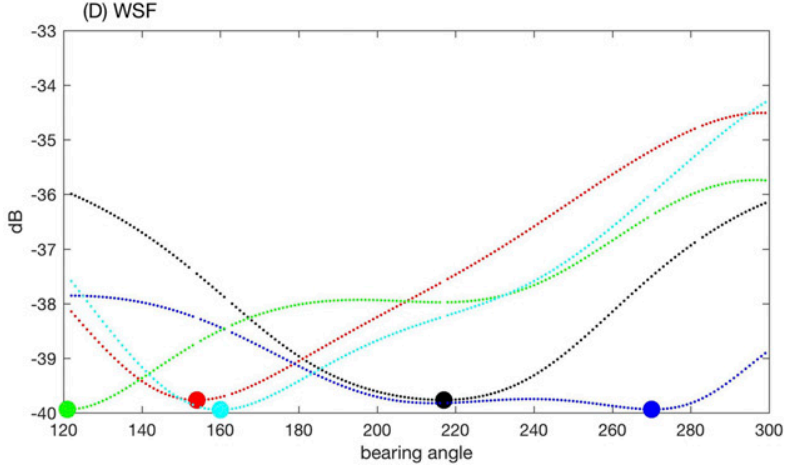

FIG. 2. Examples of the (a) $D_{\text {AIC }}$ and $D_{\text {MDL }}$ emitter number estimates, (b) the MUSIC DOA function (i.e., $P_{\text {MUSIC }}$ ) for an assumed 5 emitter solution, and (c) $P_{\mathrm{MLE}}$ and (d) $P_{\mathrm{WSF}}$ for each emitter of the assumed 5 emitter case. In each case, the found emitters are marked with circles at the maxima (or minima for from the local minimum of the detection score. A maximum of $N=5$ is used here to limit potentially spurious solutions when local minima are not found in $D_{\text {AIC }}$ or $D_{\mathrm{MDL}}$

\section{2) EMPIRICAL DETECTION METHODS}

In contrast to these statistical approaches, an empirical method for determining the number of emitters was developed for the Coastal Ocean Dynamics Application Radar (CODAR) SeaSonde (Lipa et al. 2006). This methodology uses a set of empirical parameters to compare the relative magnitudes of the eigenvalues, $\lambda$, and components of the signal power matrix, $\mathbf{P}$, estimated within MUSIC, to determine the number of emitters present. From Schmidt (1986), the $N \times N$ matrix $\mathbf{P}$,

$$
\mathbf{P}=\left(\mathbf{A}^{H} \mathbf{A}\right)^{-1} \mathbf{A}^{H}\left(\mathbf{C}-\lambda_{\min } \mathbf{I}\right) \mathbf{A}\left(\mathbf{A}^{H} \mathbf{A}\right)^{-1},
$$

where $\lambda_{\min }$ is the smallest signal eigenvalue assumed, describes the signal power and cross products for the assumed number of emitters present, here $N$. Specific to the SeaSonde, which uses a 3-antenna receive array and can sense up to two emitters (Barrick and Lipa 1999; Lipa et al. 2006), the ratios of the eigenvalues, the diagonal elements of $\mathbf{P}$, and the off-diagonal elements of $\mathbf{P}$ are compared for the two emitter (aka dual angle) solution. If any of these three ratios exceeds a threshold value, set by inspection, the two emitter solution is rejected in favor of the single emitter solution.

This method can be generalized for arbitrary receive antenna arrays using two additional steps: 1) The MUSIC DOA solution for an assumed $N$ emitters are the $\theta$ found at peak values of $P_{\text {MUSIC }}$. However $P_{\text {MUSIC }}$ (e.g., Fig. 2b) may or may not have $N$ distinct peaks, as a peak-finding algorithm is normally used to independently identify the local maxima of $P_{\text {MUSIC. }}$ As seen in the simulation-based analysis of Laws et al. (2000), estimating $P_{\text {MUSIC }}$ for all values of $1<N<M-1$ and identifying those where the predicted number of peaks matches the observed number of peaks found in $P_{\text {MUSIC }}$ offers a viable method for identifying potentially appropriate values for $N$ (Fig. 2). This step is dependent on a threshold used within the peak-finding algorithm, but can greatly reduce the potential solutions for large receive antenna arrays. 2) DOA solutions passing step 1 can then be compared sequentially

WSF) of the functions. By definition, $P_{\mathrm{MLE}}$ and $P_{\mathrm{WSF}}$ will always return the same number of emitters as requested; however, the number of emitters found in $P_{\text {MUSIC }}$ depends on an additional peak-finding step to identify the local maxima present. 
TABLE 1. HF radar data collection parameters.

\begin{tabular}{lc}
\hline \multicolumn{1}{c}{ Parameter } & Setting \\
\hline Signal modulation & FMCW linear sweep \\
Sweep rate & $\sim 0.34 \mathrm{~s}$ \\
Rx antennas & 8 \\
Rx antenna type & $\lambda / 8$ length active nonresonant monopoles \\
Rx antenna configuration & Modified rectangular array with $\lambda / 2$ diagonal spacing and one corner element removed \\
Tx antenna type & $\lambda / 4$ length passive resonant monopoles with buried ground plane \\
Tx antenna configuration & Phased array, either quad or pair \\
Data collection interval & 29.5 min \\
Raw time series file sample rate & $\sim 12 \mathrm{kHz}$ \\
Raw time series file size & $\sim 2.5 \mathrm{~Gb}$ \\
Decimated time series sample rate & $\sim 380 \mathrm{~Hz}$ \\
Decimated time series file size & $\sim 16 \mathrm{Mb}$ \\
Center transmit frequency & $\sim 16.15 \mathrm{MHz}$ \\
Transmit bandwidth & $100-75 \mathrm{kHz}^{\mathrm{a}}$ \\
Range resolution & $1.5-2 \mathrm{~km}^{\mathrm{a}}$ \\
\hline
\end{tabular}

${ }^{\text {a }}$ Transmit bandwidths were reduced to $75 \mathrm{kHz}$ in April 2018 to avoid overlap of the simultaneously transmitting radars, altering the range resolution to $2 \mathrm{~km}$.

following Lipa et al. (2006) utilizing the ratios of the eigenvalues, the diagonal elements of $\mathbf{P}$, and the offdiagonal elements of $\mathbf{P}$ against the MUSIC threshold values to reject or accept the solution with the higher number of peaks. For example, if solutions for $N=1-3$ each have the correct number of peaks, the three ratios defined above for $N=2$ are used to choose between the $N=1$ and $N=2$ solutions, similar to Lipa et al. (2006), and the ratios for the $N=3$ solution are used to choose between $N=3$ and the "winner" of the $N=1$ versus $N=$ 2 solution comparison. This generalized implementation of Lipa et al.'s (2006) method is referred to as the "SeaSonde" method below.

However, it is suggested here that step 1, as described above, exerts such a strong control on eliminating incorrect solutions that a more streamlined empirical method could simply identify the highest $N$ where the observed number of peaks match that predicted. Referred to here as the "MUSIC-highest" method, this simplification eliminates the need for the three MUSIC thresholds used by Lipa et al. (2006), but makes the threshold value used to identify a local maximum in DOA as a "peak" a key component of evaluating $P_{\text {MUSIC }}$ for multiple emitters. Here, peaks are defined as local maxima that exceed the background values of the logarithm of the DOA function by a threshold value (i.e., Fig. 2b). Threshold values of $0.05,0.25$, and 0.5 are tested below.

Critically, neither of these empirical approaches for estimating $N$, based on the DOA function, can be applied to MLE- or WSF-type DF methods without negating their potential benefit over MUSIC. Any advantages in using MLE-based methods derive in part from their ability to perform in signal conditions that compromise the effective resolution of the DOA function (Ziskind and Wax 1988; Emery 2018). Thus, all four emitter detection methods (AIC, MDL, SeaSonde, and MUSIC-highest) were used with MUSIC to estimate the radial velocities, but only the AIC and MDL methods were used with the MLE and WSF direction finding algorithms. Each of these combinations were assessed using the observations described below.

\section{HF radar implementation and data processing}

Two 8-channel HF radars developed at the University of Hawai'i (described in appendix A) were deployed on the islands of Nantucket (NWTP; $41.2^{\circ} \mathrm{N}, 70.1^{\circ} \mathrm{W}$ ) in June 2017 and Martha's Vineyard (LPWR; $41.3^{\circ} \mathrm{N}, 70.7^{\circ} \mathrm{W}$ ) in April 2018, to observe the small-scale current structures and strong tidal variability known to exist over the New England shelf (Shearman and Lentz 2004; Wilkin 2006; Kirincich and Lentz 2017) at the highest spatial resolution possible. Both systems (Table 1) transmitted in the 16.1-16.2 MHz ITU frequency band (wavelengths of $\lambda=18.6 \mathrm{~m}$ ) and were configured as arrays of $M=8$ receive antennas on a $3 \times 3$ square grid, with one corner removed, having an element spacing of $\lambda /(2 \sqrt{2})$, or $\lambda$ across the diagonal of the grid.

The complex-demodulated received signals were lowpassed filtered for antialiasing and decimated on-site to a sampling frequency of $\sim 380 \mathrm{~Hz}$ (allowing a maximum range of $120 \mathrm{~km}$ ), recorded as 30-min compressed time series, and uploaded to a central server at WHOI for postprocessing. Each 30-min data collection window was processed independently, with no temporal averaging or smoothing performed across adjacent windows. 
TABLE 2. Data processing parameters varied.

\begin{tabular}{lc}
\hline \multicolumn{1}{c}{ Parameter } & Values/methods used \\
\hline Direction finding methods & MUSIC, MLE-AP, WSF \\
Detection method & AIC, MDL, MUSIC SeaSonde, ${ }^{\mathrm{a}}$ \\
& MUSIC highest \\
Doppler spectra length & $512,1024,2048$ \\
Spectral overlap percentage & $50 \%$ \\
Covariance matrix ensemble & $16,9,4$ \\
members & \\
\hline
\end{tabular}

${ }^{a}$ Follows Lipa et al. (2006) to use empirical tests to define the emitter number.

${ }^{\mathrm{b}}$ Created as a result of the present effort as explained in the text.

For each window, the data was processed by (i) performing range-resolving FFTs, (ii) estimating individual Doppler spectra over $K$ time series segments (or snapshots) extracted from each time window, and (iii) ensemble-averaging $K$ individual Doppler autoand cross-spectra to estimate $\mathbf{C}$ (Table 2). Given C, radial velocity products were estimated based on combinations of direction finding and emitter detection methods (Table 2) to produce data structures defined by the HFR_Progs MATLAB toolbox (Kaplan and Largier 2006; Kirincich 2017a) that include radial metrics output (Kirincich et al. 2012), spatially averaged radials, and NOAA Integrated Ocean Observing System (IOOS)accepted output file formats. A version of the full MATLAB-based processing package used here is available online (Kirincich 2019).

For HF radar observations, the Doppler velocity resolution is controlled by the integration time of the Doppler spectral estimate and the sweep rate of the radar. SeaSondes typically use 1024 point FFTs with a $2 \mathrm{~Hz}$ sampling rate, producing $\sim 2 \mathrm{~cm} \mathrm{~s}^{-1}$ resolution in radial velocity. Other FFT lengths are also commonly used; for example, WERA systems sample at $\sim 4 \mathrm{~Hz}$ with 2048 point FFTs (Martinez-Pedraja et al. 2013), producing $\sim 1 \mathrm{~cm} \mathrm{~s}^{-1}$ resolution (Kirincich et al. 2012; Forget 2015). Radar operators can potentially adjust any of these operational parameters to increase Doppler resolution, which increases the spatial density of radial observations and potentially decreases occurrences of multivalued solutions that exceed the sensing ability of the radar. However, in practice, there are real trade-offs between range, accuracy, and the data sampling time.

Illustrating these trade-offs, various Doppler-resolving FFT lengths were evaluated, with 1024-point $(5.5 \mathrm{~min})$ segments and $50 \%$ segment overlap proving superior as it yields $K=9$ independent spectral estimates from a single 30-min time series. Using longer 2048-point segments with $50 \%$ overlap would result in ensemble sizes of only $K=4$ and therefore, rank-deficient covariance matrices that violate the requirements of most DF methods
(Tuncer and Friedlander 2009). Using 512-point segments potentially provide a stabler result, with $K=17$, but the coarser resolution of Doppler spectra $\left(\sim 5 \mathrm{~cm} \mathrm{~s}^{-1}\right)$ led to fewer radial results, despite more multiemitter solutions. Thus, in the remainder of this work, Dopplerresolving spectra will be based on 1024-point segments (as shown in Fig. 3).

DF algorithms incorporate both the physical spacing of receive antennas as well as amplitude and phase differences in antenna, filter, cable, and hardware responses that cause departures from the response of an "ideal" array through the measured antenna pattern, or manifold [A in Eq. (1), etc.]. Receive array antenna patterns, as well as the relative phase errors, were measured at both sites using both an independent local source (Washburn et al. 2016) and by conducting bistatic tests between the two systems as described in appendix B. The analysis performed here uses ideal antenna patterns covering just the overwater portion of the antenna bearings, versus full $360^{\circ}$ patterns, which were not found to lead to statistically different results for any test.

Data from two separate periods were used to make methodological comparisons. The first period was a 2.5-day time period from the NWTP system only, which coincided with a mass surface drifter release within the coverage of the radar on 15-17 August 2017. In total, 20 standard Coastal Dynamics Experiment (CODE)-style (Davis 1985) surface drifters with a maximum drogue depth of $1-\mathrm{m}$ were launched within a $5-\mathrm{km}$ by $5-\mathrm{km}$ area over a period of $3 \mathrm{~h}$ and allowed to advect out of the radar coverage domain (Fig. 4). Drifter trajectories were converted to Eulerian velocities, spatially averaged over nonoverlapping $5^{\circ}$ azimuthal bins along each range circle, and projected into radial directions toward the radar to form independent time series of radial velocities at each radial grid point for direct comparison (Fig. 5a).

The second time period examined was a 14-day period in August 2018, where both 16-MHz sites (LPWR and NWTP) were operating as well as two $25 \mathrm{MHz}$ SeaSonde's located on the western and eastern sides of Martha's Vineyard [sites METS and SQUB as described in Kirincich and Lentz (2017)]. The 25-MHz sites were used to produce vector velocities of the surface currents using commonly employed methods (Kaplan and Largier 2006) and then transformed into a coordinate system aligned with either of the UH radars, producing an independent estimate of radial velocity based on the $25-\mathrm{MHz}$ data (hereafter "synthetic radials"). Given the placement of the higher-frequency sites, this second analysis period enabled comparisons of synthetic and observed radials along multiple range circles of the UH radar systems for the 14-day period. While only a subset 

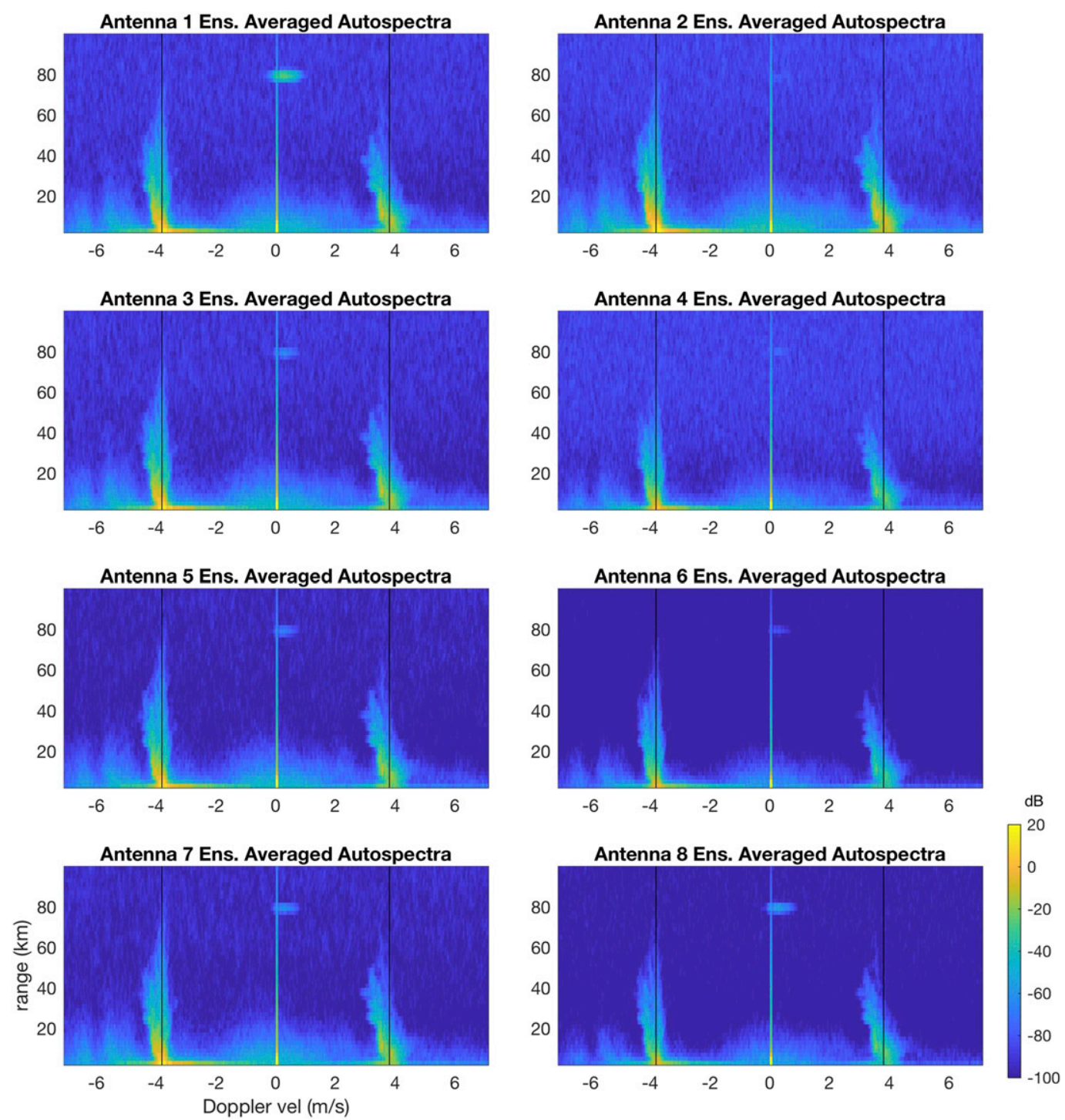

FIG. 3. Auto-spectra from all 8 channels of the NWTP radar site collected at 1700 UTC 23 May 2018 in (arbitrary referenced) dB.

of the potential methodological comparisons are shown for this time period, improvements in comparison statistics [e.g., correlation coefficient and root-mean-square difference (RMSD)] between the synthetic and measured radials indicate improved performance of one method over another.

\section{Results}

A sample application of the direction finding and detection methods (Fig. 2: based on a covariance matrix from the spectra in Fig. 3) illustrates the potential differences among the detection and DF methods. In this example, $D_{\mathrm{MDL}}$ and $D_{\mathrm{AIC}}$ from Eqs. (6) and (7) (Fig. 2a) have no local minima, and thus, the maximum number of emitters allowed, $N=5$, were assumed. By design, both the MLE and WSF methods return solutions for all predicted emitters (Figs. 2c,d). These locations are marked by peaks (for MLE) or troughs (for WSF) of the individual emitter response functions. Note that these functions are not equivalent to $P_{\text {MUSIC }}$, which has all potential emitters within the same function. Using $N=5$ in MUSIC gives a $P_{\text {MUSIC }}$ with two, not five, local peaks (Fig. 2b). In the August 2017 NWTP dataset, MLE and WSF methods defaulted to the maximum number of emitters $\sim 50 \%$ of the time for $N_{\max }=5$ and $\sim 80 \%$ of the time for $N_{\max }=4$. This example illustrates both that the MLE and WSF methods themselves provide no indication of the most appropriate value of $N$ and that AIC and MDL often overestimate $N$. 


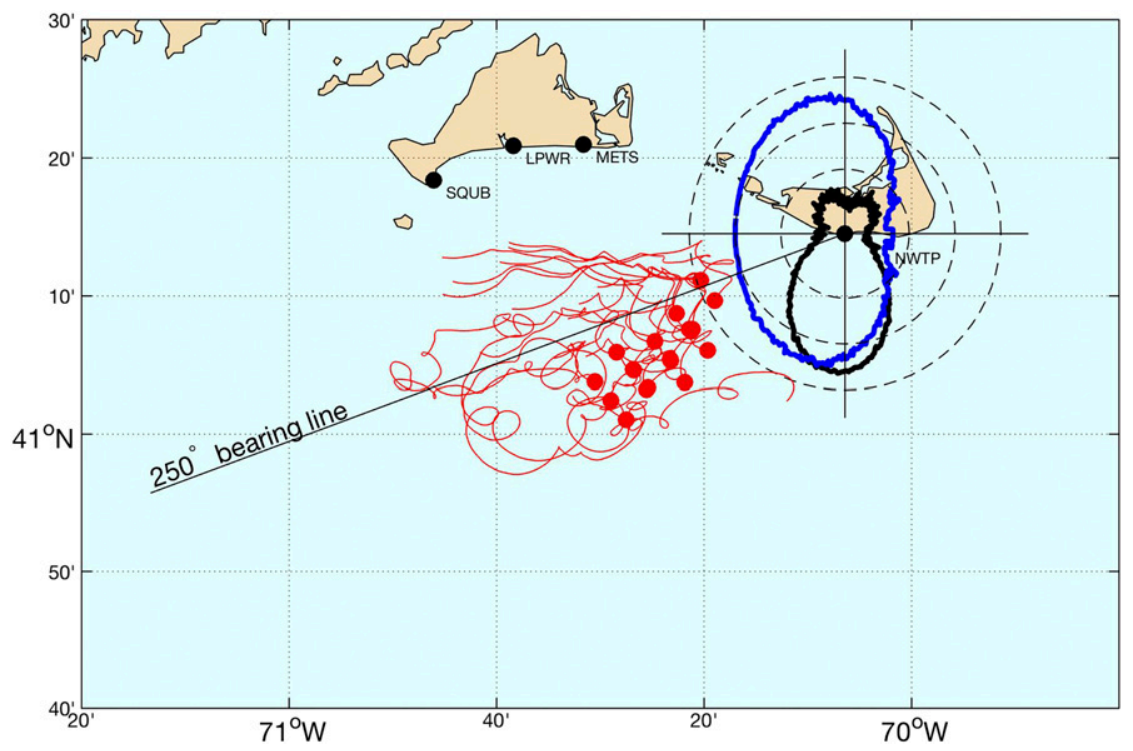

FIG. 4. Location of the radar sites on the islands of Martha's Vineyard (sites SQUB, LPWR, and METS) and Nantucket (site NWTP), Massachusetts. An overlay at the location of NWTP, illustrates the theoretical azimuthal dependence of the 2017 (black) and 2018 (blue) Tx antenna patterns, constructed assuming 5\% Gaussian random phase and amplitude noise, and shown with $5 \mathrm{dBm}$ isolines (dashed). Trajectories from the 2.5 day drifter release starting on 15 Aug are shown in red and the $250^{\circ}$ bearing line to the radar, described in the text is shown in black.

To document the relative performance of each method using the 8-antenna arrays, spectral observations from NWTP during the August 2017 test period were processed using all combinations of the three DF methods, the AIC and MDL detection tests for MLE and WSF, all four detection tests for MUSIC, and a range of FFT lengths and data snapshots (Table 2). No effort was made to test data spans longer than the 30-min native time series file, thus maximizing the temporal resolution to observe changes in the flow field.

\section{a. 2017 drifter comparisons}

For each individual radar-to-drifter velocity comparison, time series of drifter and radar radial velocities over the 2.5-day period were compared only when more than 10 independent data pairs existed for a particular location (Fig. 5a). For all methods considered, RMS differences increased from 5 to $10 \mathrm{~cm} \mathrm{~s}^{-1}$ at the southern end to more than $20 \mathrm{~cm} \mathrm{~s}^{-1}$ at the northern end of the drifter area (Fig. 5). As this pattern is independent of method within the 2017 comparisons, we hypothesize that this spatial gradient was due in part to the rapid decrease in the transmit power with azimuth moving north (i.e., $\theta>250^{\circ}$ in Fig. 4). A large decrease (i.e., $10 \mathrm{dBm})$ in transmitted power in the western part of the coverage area may cause signals from these headings to fall below the noise level, limiting the data from these azimuths.
Holding the FFT length, antenna pattern, and snapshot number constant, the relative performance of each available combination of direction finding and detection methods were estimated for the 2017 NWTP dataset. Performance was assessed in terms of the RMS difference and correlation coefficient against the in situ drifter-based velocities (Table 3 and Fig. 6). Results are shown for all data comparisons, as well as for data within the region of higher transmitted power only (radial bearings of $\theta<250^{\circ}$ ). Given a fixed detection method such as MDL or AIC, only small differences existed between each DF method, with WSF performing slightly better in RMSD but slightly worse than MUSIC in correlation. Limited to $\theta<250^{\circ}$, RMSDs decreased for MLE and WSF relative to MUSIC as did correlations. However, for all comparisons using AIC or MDL to identify the number of emitters present, the results were poor in contrast to the MUSIC results that used either of the SeaSonde or MUSIC-highest detection methods. Using MUSIC with either of these two methods, RMSDs decrease from $17-18$ to $14 \mathrm{~cm} \mathrm{~s}^{-1}$ as the MUSIC DOA threshold value was increased from 0.05 to 0.25 to 0.5 . Correlation coefficients were also higher than any DF method using MDL or AIC, with values increasing from 0.6 to 0.7 with increasing DOA threshold value. As would be expected, increasing the DOA peak threshold acts to decrease the total number of solutions returned, but as weaker peaks appear 

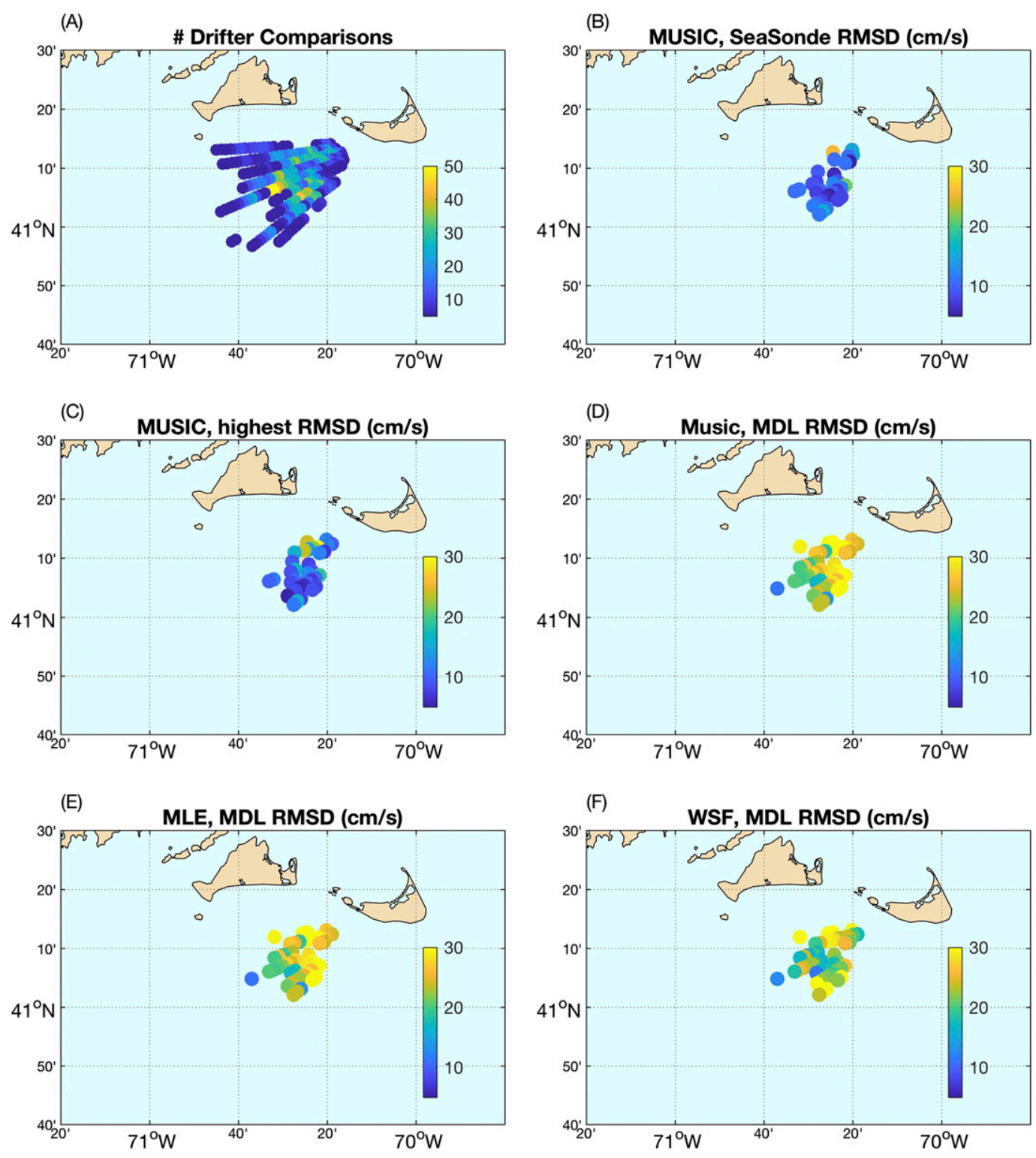

FIG. 5. (a) Drifter to radar comparison locations and sample numbers for the 2017 drifter dataset. (b)-(f) Spatial RMSD results, in $\mathrm{cm} \mathrm{s}^{-1}$, for a range of the methods examined here.

to be more uncertain (Emery and Washburn 2019), the higher threshold is able to significantly improve the comparisons with the drifter-based velocities. Limiting to $\theta<250^{\circ}$ improves the comparisons further, decreasing RMSDs to $11 \mathrm{~cm} \mathrm{~s}^{-1}$ and increasing correlations to 0.83 for a DOA threshold of 0.5. At higher values of DOA threshold, there was not a significant difference in the performance of the SeasSonde detection method against the simpler MUSIC-highest method (Table 3 and Fig. 6).

\section{b. 2018 synthetic radial comparisons}

The 2017 comparison using NWTP radials and drifters demonstrated that the MUSIC-highest processing methodology appeared to be the most useful, given its combination of high correlation and low RMSD with the least number of tunable parameters. However, those comparisons were limited to a relatively small azimuthal extent of the radar due to the location of the drifter deployment relative to the radar's transmitted power. In 2018, the NWTP transmit beam pattern was adjusted to provide increased energy levels to the west to mitigate this issue (Fig. 4) and data from the second site, LPWR became available. To confirm improvements in the azimuthal extent of the transmit power at NWTP, and examine any additional azimuthal dependence of either system, radial velocities based on the MUSIC-highest 
TABLE 3. Methodological comparisons using NWTP data against the August 2017 drifter release.

\begin{tabular}{|c|c|c|c|c|c|c|}
\hline \multirow[b]{2}{*}{ DF } & \multirow[b]{2}{*}{ Emitter ID } & \multirow[b]{2}{*}{ Peak threshold } & \multicolumn{2}{|c|}{ All data } & \multicolumn{2}{|c|}{ Southern portion } \\
\hline & & & $\operatorname{RMSD}\left(\mathrm{m} \mathrm{s}^{-1}\right)$ & $\overline{\mathrm{CC}}$ & $\operatorname{RMSD}\left(\mathrm{m} \mathrm{s}^{-1}\right)$ & $\mathrm{CC}$ \\
\hline MUSIC & MDL & 0.5 & 0.27 & 0.50 & 0.27 & 0.53 \\
\hline MLE & MDL & $\mathrm{n} / \mathrm{a}$ & 0.27 & 0.50 & 0.27 & 0.53 \\
\hline WSF & MDL & $\mathrm{n} / \mathrm{a}$ & 0.26 & 0.46 & 0.25 & 0.35 \\
\hline MUSIC & AIC & 0.5 & 0.26 & 0.51 & 0.26 & 0.55 \\
\hline MLE & AIC & $\mathrm{n} / \mathrm{a}$ & 0.28 & 0.46 & 0.23 & 0.47 \\
\hline WSF & AIC & $\mathrm{n} / \mathrm{a}$ & 0.25 & 0.49 & 0.23 & 0.37 \\
\hline MUSIC & SeaSonde & 0.05 & 0.17 & 0.63 & 0.13 & 0.76 \\
\hline MUSIC & SeaSonde & 0.25 & 0.14 & 0.70 & 0.11 & 0.80 \\
\hline MUSIC & SeaSonde & 0.5 & 0.13 & 0.73 & 0.11 & 0.82 \\
\hline MUSIC & Highest & 0.05 & 0.18 & 0.59 & 0.14 & 0.74 \\
\hline MUSIC & Highest & 0.25 & 0.15 & 0.67 & 0.12 & 0.79 \\
\hline MUSIC & Highest & 0.5 & 0.14 & 0.72 & 0.11 & 0.83 \\
\hline
\end{tabular}

method were estimated from both 8-channel systems and compared with the available data from two $25 \mathrm{MHz}$ SeaSondes that operated with higher spatial resolution.

Data from both LPWR and NWTP in August 2018 show reasonable agreement with time series of synthetic radials constructed from the $25-\mathrm{MHz}$ sites (SQUB and METS). The effect of the single parameter in the MUSIC-highest method, the DOA peak threshold, was estimated using values of 0.25 and 0.5 , respectively. For NWTP, the RMSDs along 3 range circles that overlap the vector coverage area (Fig. 7) were between 18 and $11 \mathrm{~cm} \mathrm{~s}^{-1}$ and tended to decrease southward. The 2018 comparisons show a marked improvement over the 2017 tests, despite being well north of the $\theta=250^{\circ}$ bearing line (Fig. 4). For LPWR, a larger $\sim 100^{\circ}$ azimuthal span was covered by the synthetic radials. RMSDs for 3 representative range cells (Fig. 7) varied between 18 and $10 \mathrm{~cm} \mathrm{~s}^{-1}$ with azimuth, with an overall mean of $\sim 13 \mathrm{~cm} \mathrm{~s}^{-1}$. The cause of increased error for range cell 7 between bearings $130^{\circ}$ and $150^{\circ}$ is not clear. In general, increasing the MUSIC DOA peak threshold (dashed lines) decreased the RMSD by up to $2 \mathrm{~cm} \mathrm{~s}^{-1}$. Varying other parameters (i.e., Table 2) or using other methodologies gave similar results to those described above for the drifter-based comparisons, and are not shown here.

\section{Discussion}

Improving the ability of HF radars to resolve two or more independent signals that may be closely spaced in bearing or Doppler velocity is critical to accurately sensing complex coastal flows. This work advances the ability of HF radars to measure complex flows by both changing how radars themselves are configured and improving how currents are extracted from radar observations. Using observations from 8-antenna $\mathrm{HF}$ radar systems, configured as rectangular phased arrays but operated as direction finding systems, the tests performed here suggest that DOA estimates using the MUSIC algorithm and the MUSIC-highest emitter detection method with a peak threshold of 0.5 provide a robust method to estimate surface currents with the lowest number of tunable parameters. The accuracy of the combined radar configuration and bearing-determination method are reasonable in comparison to previous analyses (e.g., Kohut et al. 2006; Ullman et al. 2006; Ohlmann et al. 2007; Kirincich et al. 2012) given the high spatial and temporal independence of the data. For example, the RMSDs of Figs. 5b and 5c are comparable to those reported by Ohlmann et al. (2007, see their Table 3). These tests also demonstrate that the 8-antenna arrays yield surface currents with increased azimuthal resolution, given their ability to detect more radial vectors at a given Doppler velocity within a range circle. During the 14-day sample period in August 2018, only $18 \%$ of the estimated radial velocity solutions were shown to be singleemitter solutions (Fig. 8), 56\% were found to be twoemitter solutions, and $\sim 26 \%$ of the solutions had either three or four emitters. Thus, a quarter of the spectral estimates submitted to the direction finding algorithm would be incorrectly resolved by a standard 3-antenna SeaSonde configuration. This improvement in the azimuthal resolution of radial currents is likely to improve observations of small-scale current structures and reduce errors. The poor performance from the AIC and MDL detection methods and the justification for using the simpler MUSIC-highest detection method are discussed in more detail below.

Signal processing applications for the AIC and MDL methods typically have spatially white noise (Krim and Viberg 1996), distinct breaks in magnitude of the eigenvalues of the covariance matrix (Johnson and Dudgeon 1993), and a multiplicity of roughly equal noise eigenvalues (Viberg et al. 1991). Thus, both AIC and MDL depend on 

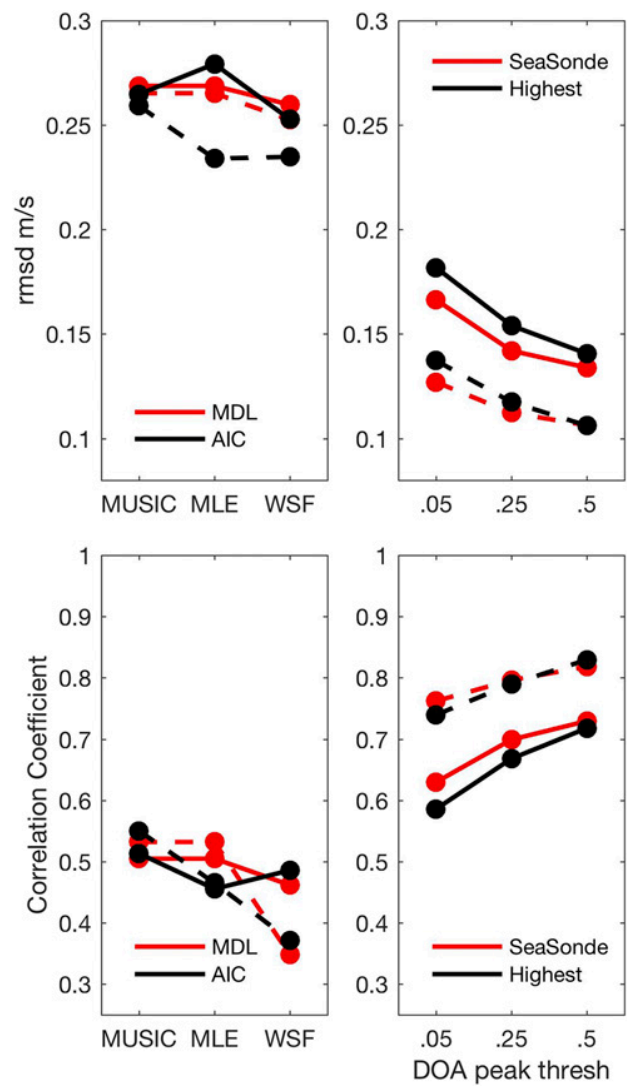

FIG. 6. Root-mean-square differences (RMSD) and correlation coefficients (CC) between NWTP radial velocities and drifterbased radial velocities from August 2017 (Table 3). All (left) DF methods and (right) MUSIC peak thresholds are compared for all data (solid lines) and only data with radial bearings $\theta<250^{\circ}$ (dashed) (Fig. 4), to eliminate the poor quality returns at the northern end of the domain.

having a distinct separation between the signal eigenvalues and the noise eigenvalues-a characteristic not typically found in the eigenvalues of oceanographic data. As illustrated by the example in Fig. 8, the sorted eigenvalues of oceanic radar backscatter typically lack a well-defined break separating signal and noise. A similar continuous rolloff was seen by Emery (2018) for simulations using the high-resolution numerical output shown in Fig. 1. Under these conditions, statistical tests such as AIC and MDL overpredict the emitter number, leading to spurious DOA solutions. This analysis limited the maximum allowable number of emitters to $N=5$, a limit that was often reached with either AIC or MDL.

Figure 2 illustrates an example of the effect of the statistical overprediction of emitters on each of the DOA methods, as both AIC and MDL defaulted to the maximum number of emitters allowed. Although the five emitter solution is shown for MUSIC, only two distinct peaks were found in the DOA (Fig. 2b).

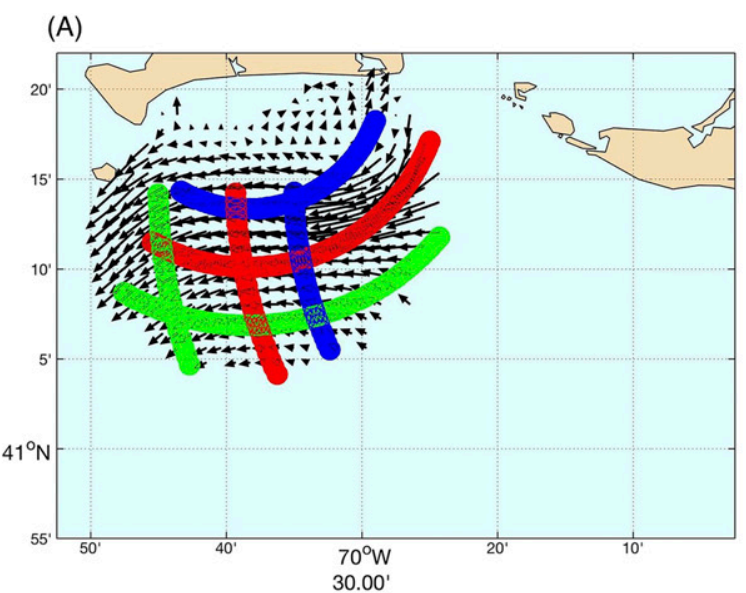

(B)

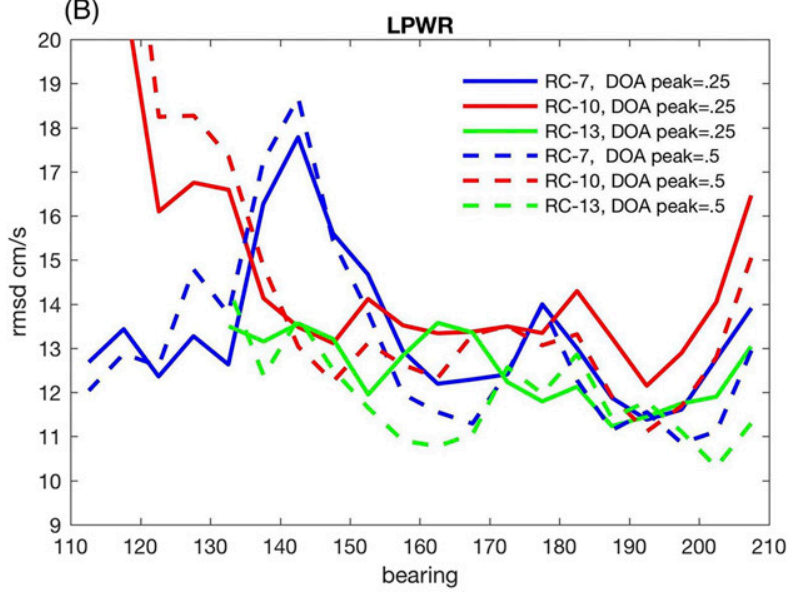

(C)

NWTP

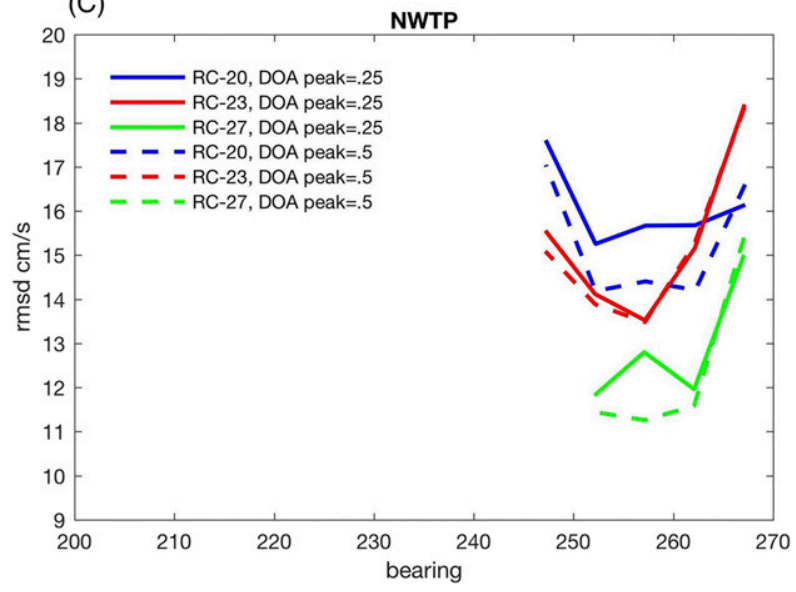

FIG. 7. (a) Mean surface currents from 5 to 19 Aug 2018, formed using 25-MHz SeaSonde sites, SQUB and METS, with the range circles used to construct synthetic radials comparisons marked. (b) RMSD for LPWR radials formed using the MUSIC-highest emitter detection method with DOA peak thresholds of 0.25 (solid) and 0.5 (dashed) against synthetic radials, formed from SQUB and METS as described in the text for range circles 7, 10, and 13. (c) Same for NWTP radials for range circles 20, 23, and 27 . 

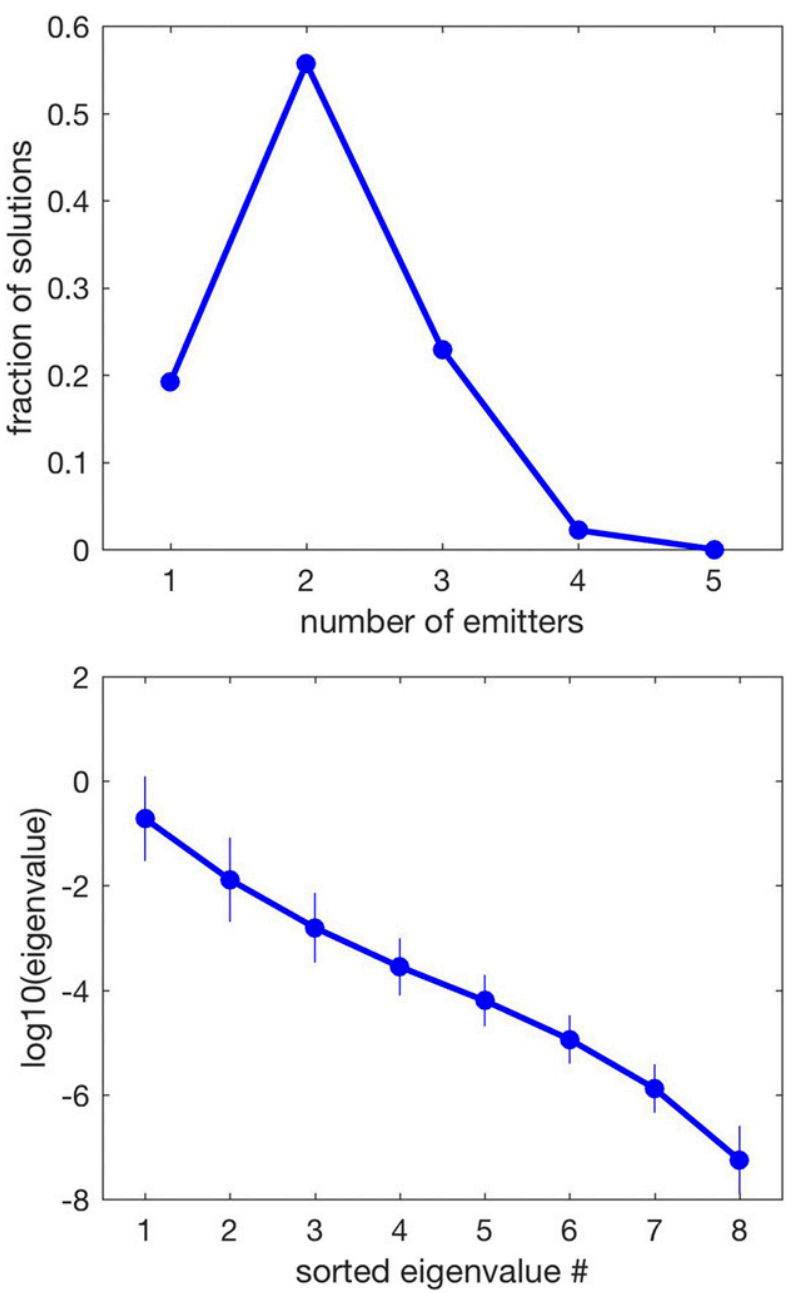

FIG. 8. (top) Statistics of the emitter number and (bottom) mean sorted eigenvalues with standard deviation for LPWR range cell 7 , as shown in Fig. 7, for the 14 day period in August 2018.

Within the MLE results, four of the predicted emitters are within $20^{\circ}$ of one of the MUSIC DOA peaks, while WSF-predicted emitters are more evenly spread in bearing, with three located at or adjacent to the MUSIC DOA peaks (Figs. 2c,d). Both methods have additional solutions that do not directly correspond to any of the observed MUSIC DOA peaks. The tightly spaced solutions for MLE and WSF are closer than MUSIC could resolve (Tuncer and Friedlander 2009), but the spurious results from MLE and WSF solutions would result in higher radial velocity errors, as was shown above. In the example of Fig. 2b, representative of most solutions found using AIC or MDL to estimate the emitters present, the observed number of peaks in the MUSIC DOA was generally less than the number predicted. However, it should be noted that, using AIC or MDL for detection, MUSIC does no better than MLE or WSF in independent comparisons (Fig. 6) and thus the DOA peak-finding step does not itself offer an improvement over alternative methods.

Both the parametric approach, following Lipa et al. (2006), as well as the simplified MUSIC-highest approach performed better likely because the additional criteria requiring the observed number of MUSIC DOA peaks to match that predicted for the solution. The MUSIC solution shown in (Fig. 2) would not be chosen using either the SeaSonde or MUSIC-highest method. In this example eigenvalue solution, only the 1 and 3 emitter solutions for MUSIC satisfy the peak number criteria (Fig. 9, left). In a second example from the same data file, only the 1 and 2 emitter solutions satisfy the criteria. Thus, this criterion alone limits the potential solutions and plays a singular role in making MUSIC the superior DF method in this analysis (Fig. 9, right).

Contrasting the results between the SeaSonde and MUSIC-highest emitter methods, when any DOA peak is taken as an emitter by using a small peak threshold, the SeaSonde parametric approach has reduced errors. By using the three threshold tests described by Lipa et al. (2006), SeaSonde effectively looks for an equivalent break in magnitude of the signal and noise components of the covariance matrix using more information than the AIC or MDL methods. As the ratio of the eigenvalue magnitude test as well as the test based on the estimate of the relative signal powers [Eq. (8)] are roughly equivalent to an AIC/MDL approach of looking for a break in the eigenvalues, it is the off-diagonal ratio test (Lipa et al. 2006) that most often steers the results. In the sample data file highlighted here (Fig. 3), this third criterion is exceeded $90 \%$ of the time when the higher emitter solution is rejected. When the DOA peaks are chosen using a larger peak threshold, there is no significant difference between the SeaSonde approach and simply picking the highest viable solution, as is done in MUSIChighest. Using a higher peak threshold effectively reduces the total number of emitters detected, and those emitters with low-magnitude peaks appear to have higher errors in their azimuthal placement than highmagnitude peaks.

The correspondence between SeaSonde and MUSIChighest detection methods at increased peak thresholds brings up a fundamental difference between the standard 3-channel SeaSonde antenna and the 8-channel systems used here. Experience using the 3-antenna SeaSonde with MUSIC-highest detection indicates that two DOA peaks can nearly always be found when searching for two emitters. That is, the MUSIC-highest method fails and a parametric approach (SeaSonde) is required for accurate detection. Why then is the MUSIC-highest detection method as good as SeaSonde with 8 antennas? The answer is likely related to how MUSIC fundamentally 

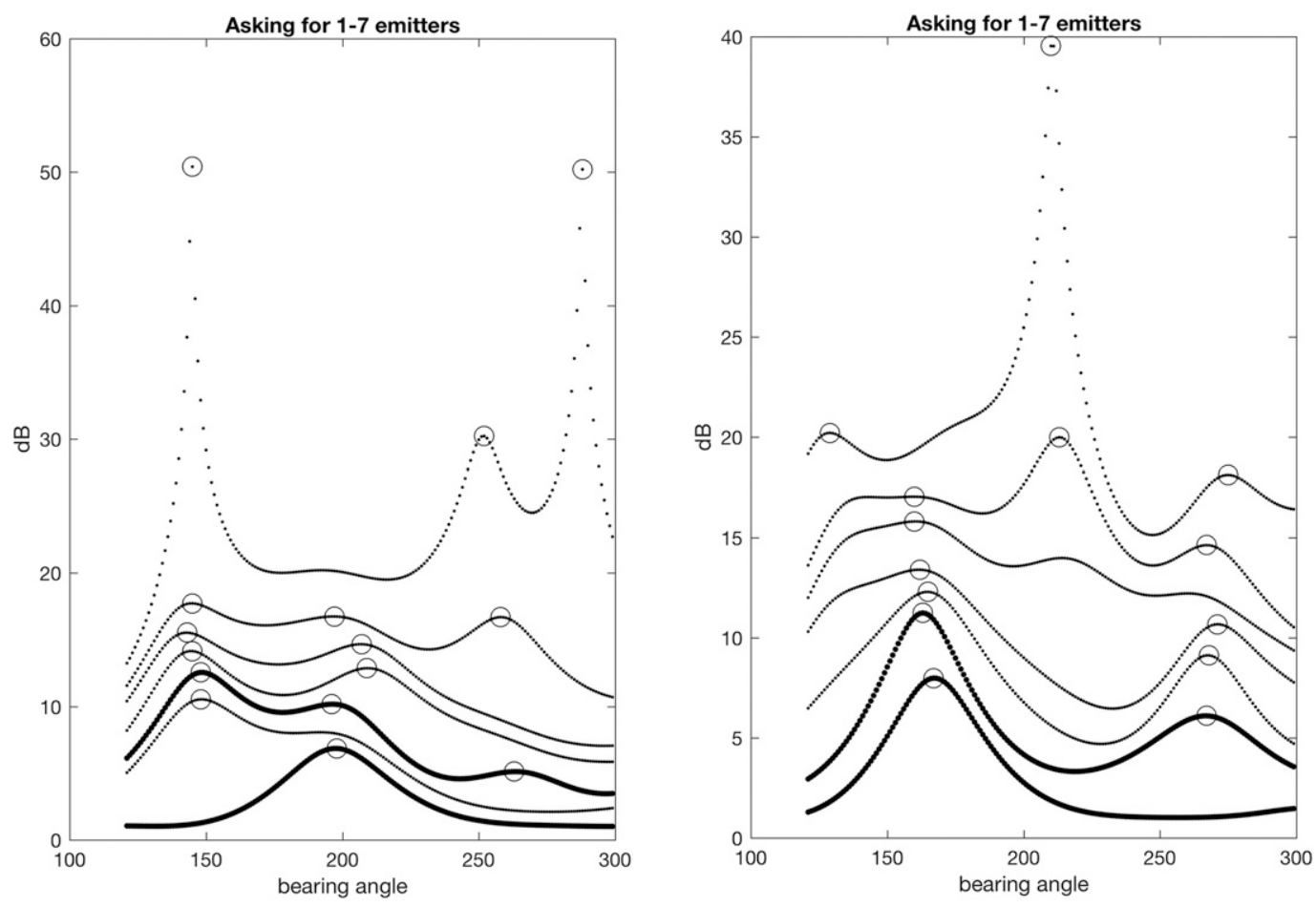

FIG. 9. Sample estimates of the MUSIC DOA for (left) the same eigenvalue solution used in Fig. 2 and (right) a second example from the same data file. For each, all potential emitter numbers between 1 and 7 , offset vertically starting with the one emitter solution at the bottom are shown. Distinct peaks for each case are marked with open circles. Cases where the number of predicted emitters matched those observed are shown in bold. Using the MUSIC-highest detection method, the three and two emitter solutions would be chosen for the left and right examples, respectively.

works: MUSIC finds the DOA solutions by using the noise subspace, and having more antennas and thus a larger number of noise eigenvalues for a given $N$ permits a more accurate determination of the noise subspace, which leads to reduced MUSIC DOA errors (Stoica and Nehorai 1989). As shown in Fig. 9, higher emitter predictions tend to return lower numbers of peaks than predicted. However, the peak locations become more variable, and potentially spurious returns are given as the predicted emitter number increases and the eigenvectors defining the noise subspace decreases. Thus, in practice one can make an incorrect prediction of the number of emitters present and still get close to the correct bearings, as long as the noise subspace is well defined by multiple eigenvectors. Exactly how many noise eigenvalues are needed is likely to vary with the covariance matrix but, limited to well-defined DOA peaks, the MUSIC-highest emitter method appears to function well for up to 4 emitters with 8 antennas.

Finally, the methodology developed here is adaptable to arbitrary configurations of the receive antenna array as well as arbitrary numbers of receive elements. While both influence the potential accuracy and azimuthal resolution of the radar, both can be easily accounted for in $\mathbf{A}$, the antenna response matrix (Tuncer and Friedlander 2009) used by each of the DOA methods to account for the placement, amplitude response, and phase lags of each antenna element within the DF calculation.

\section{Summary}

This work evaluates the performance of an 8-antenna phased-array HF radar using several direction finding methods to estimate the radial surface currents. Determining the correct number of emitters is critical for direction finding systems, and the design of the processing methods must be carefully considered to realize both accurate results and maximize the azimuthal resolution. A combination of the MUSIC algorithm and the "highest" emitter detection method had both accurate results and fewer tunable parameters. MUSIC was preferred here only because the MUSIC DOA function allows for an accurate estimate of the number of emitters. Other direction finding methods were shown to perform better than MUSIC under similar conditions; however, 


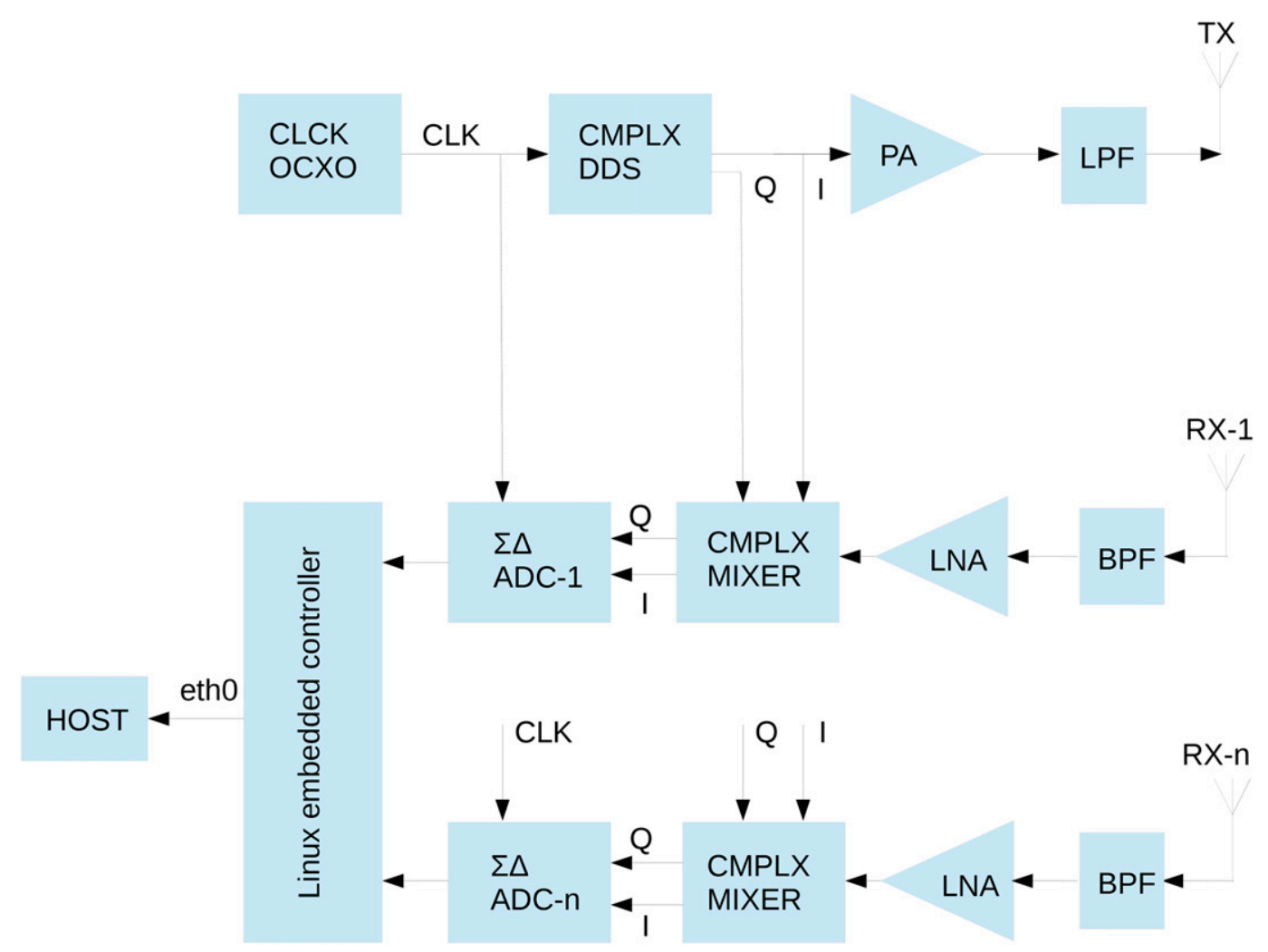

FIG. A1. Block diagram for the UH FMCW HF radar system, as described in appendix A.

these methods required alternative emitter detection techniques that performed poorly for HF radar datasets. For the 8-antenna arrays, requiring the observed number of MUSIC DOA peaks to match that predicted for the solution led to significant error reductions over the AIC or MDL detector identification methods. Further, simplistic detection methods based on the DOA output were shown to perform well for the 8-antenna systems, likely because the MUSIC noise subspace was welldefined, improving accuracy. Using the methodology described here, available as an open-source MATLAB package (Kirincich 2019), the 8-antenna systems improved observations of radial currents by sensing multivalued direction finding solutions that would otherwise be missed by standard methods.

Acknowledgments. This analysis was supported by NSF Grants OCE-1657896 and OCE-1736930 to Kirincich, OCE-1658475 to Emery and Washburn and OCE-1736709 to Flament. Flament is also supported by NOAA's Integrated Ocean Observing System through Award NA11NOS0120039. The authors thank Lindsey Benjamin, Alma Castillo, Ken Constantine, Benedicte Dousset, Ian Fernandez, Mael Flament, Dave Harris, Garrett Hebert, Ben Hodges, Victoria Futch, Matt Guanci, and Philip Moravcik for assistance in building, deploying, and operating the radars.

\section{APPENDIX A}

\section{The University of Hawai'i High-Frequency Doppler Radar}

The University of Hawai'i high-frequency Doppler radar is a generic frequency-modulated continuous wave radar based on the principle of base-band complex demodulation, aka homodyne detection (Fig. A1). A classical rack-mounted model was produced in 2012, and a compact portable model in 2017; both models were used in the present project.

In the UH radar, an oven-controlled crystal oscillator $(\mathrm{OCXO})$ provides a stable reference frequency $(100 \mathrm{MHz})$ both to the direct digital synthesizer (DDS) and to the bank of analog-to-digital converters (ADC). An Analog Devices AD9854 integrated circuit DDS is used, featuring a 48-bit tuning word with $\mu \mathrm{Hz}$ frequency resolution and dual-quadrature 12-bit digital-to-analog converters clocked at $300 \mathrm{MHz}$ through a clock multiplier. The AD9854 DDS achieves a spurious-free dynamic range better than $90 \mathrm{~dB}$, and residual phase noise 
better than $140 \mathrm{dBc} \mathrm{Hz}^{-1}$ at $1 \mathrm{kHz}$ offset (the phase noise of the OCXO is $\sim 150 \mathrm{dBc} \mathrm{Hz}^{-1}$ ). A simple uninterrupted linear sweep modulation is chosen as the transmitted wave form. The DDS signal is amplified up to $50 \mathrm{~W}$, lowpass filtered to remove spurious harmonics, and split into an array of transmit antennas, built as resonant monopoles with a buried ground plane (Table 1).

An array of receive antennas collects the echoes backscattered from the ocean. The receive antennas are active nonresonant monopoles to maintain phase stability over the sweep bandwidth. Each antenna channel is bandpass filtered to reject out-of-band high-frequency energy, amplified through a low-noise amplifier (LNA), and fed to a pair of double-balanced diode ring mixers, excited by orthogonal local oscillator signals from the DDS. This performs the complex demodulation of the received signals using a copy of the transmit signal. The complex-demodulated low-frequency signals are digitized by a bank of ADCs, and recorded on an embedded Linux computer for postprocessing.

A Texas Instrument ADS1278 integrated circuit ADC is used, featuring 24-bit sigma-delta conversion with a/512 digital finite impulse response (FIR) low-pass/decimation filter. The ADS1278 ADC achieves a signal-to-noise ratio better than $110 \mathrm{~dB}$ for high-frequency sampling at $6 \mathrm{MHz}$ and decimation to $12 \mathrm{kHz}$ or an effective number of bits (ENOB) of 19.5. A further decimation $/ 32$ down to $380 \mathrm{~Hz}$ is performed on the host computer in MATLAB using eighth-order Chebyshev low-pass filters, increasing in principle the ENOB to 24 and the dynamic range to $140 \mathrm{~dB}$. All raw receive antenna complex time series are recorded synchronously in parallel, permitting either BF and DF processing methods.

The deployment of frequency-modulated continuous wave (FMCW) radars that transmit and receive signals simultaneously (uninterrupted) requires particular antenna configurations to maximize the range, which is governed not by the total transmitted power, but by how much the direct path energy from the transmit antennas can be attenuated. In addition to the weak Bragg-scattered energy from the ocean, the first stage of the low-noise amplifier in the receivers must amplify linearly the reflected energy from the nearshore breaking waves and the direct path energy from the transmit antennas to avoid the generation of spurious harmonics and intermodulation products.

The direct path energy must be lowered to a level comparable to the nearshore reflected energy (which cannot be controlled), to maximize the overall dynamic range of the receivers and ADC. Placing the transmit antennas as far as possible from the receive antennas (10 and $16 \lambda$ were used at LPWR and NWTP, respectively) will lower the direct path return as land attenuation is typically $\sim 1 \mathrm{~dB}$ per $\lambda$. An array of transmit antennas phased to produce a null in the direction of the receive antennas and a wide beam toward ocean is also used. With proper antenna tuning, up to $30 \mathrm{~dB}$ reduction of the direct path energy can be achieved, compared to isotropic transmission.

\section{APPENDIX B}

\section{Calibration of the NWTP and LPWR Receive Arrays}

Significant effort was spent both installing the UH radar systems such that the data collected from the received array was as ideal as possible as well as measuring the azimuthal response pattern of the receive antenna array to provide confirmation. During the installation process, the relative phase error due to the receive cable length variability was determined to be $<2 \mathrm{~cm}$ for $100-\mathrm{m}$ length cables and the locational errors of the antenna placement were surveyed to be $<6 \mathrm{~cm}$ or effectively the width of the RX antenna post itself. Custom filters (from http://www.dlwc.com) were used for the receiver board bandpass filters that limited the phase error of the filters to $\pm 2^{\circ}$ phase. All these sources of error were small relative to the operational spread of the $\mathrm{RX}$ array with $\lambda /(2 \times \sqrt{2})$ element spacing or 6.5 -m given a transmit wavelength of $18.6 \mathrm{~m}$.

Two additional tests were used to confirm the RX array response with azimuth: 1) direct measurements of the response of the RX array to a mobile signal source and 2) bistatic tests using each radar as a source for the other. Direct measurements of the response of the RX array to a mobile signal source were made at both $\mathrm{UH}$ radar sites in 2018. The RX array's relative response with bearing was estimated by operating the mobile source at fixed frequency within the radar's chirp, and examining the signal properties of the identified peak in short 32-chirp Doppler spectra after adjusting the received phase differences (from the center antenna) for the circular versus plane wave geometry of the near-field location of the mobile source. This technique is commonly used to calibrate direction finding radar systems (e.g., Washburn et al. 2016). For both sites, these results led to measured RX array relative phase responses (at resolutions of $1^{\circ}$ ) that were not significantly different from an assumed ideal relative phase response given the error in the GPS positions of the mobile source and the potential Doppler spectral noise in estimates of the phase of each antenna relative to the center antenna. Second, bistatic tests of each radar system were made by setting both systems to continuous sine wave transmission and examining both the relative phase of 
each antenna as well at the MUSIC estimated bearing of the source or emitter (i.e., the other radar) over time scales of hours. These tests suggest that the relative phases were stable over time, and importantly, that the MUSIC estimated bearing of the source radar was correct to $\pm 1^{\circ}$.

Finally, a small number of the processing methodologies compared in the 2017 drifter to radar comparisons were reprocessed using the best fit measured $\mathrm{RX}$ array response pattern. Drifter to radar comparisons for these runs, using the measured response pattern were similar ( $\pm 1 \mathrm{~cm} \mathrm{~s}^{-1}$ in RMS difference) to those shown in the text. Based on these results, we utilize the ideal receive array antenna pattern in the results reported here for simplicity.

\section{REFERENCES}

Akaike, H., 1974: A new look at the statistical model identification IEEE Trans. Autom. Control, 19, 716-723, https://doi.org/ 10.1109/TAC.1974.1100705.

Barrick, D., 1972: First-order theory and analysis of MF/HF/VHF scatter from the sea. IEEE Trans. Antennas Propag., 20, 2-10, https://doi.org/10.1109/TAP.1972.1140123.

_ and B. J. Lipa, 1999: Radar angle determination with MUSIC direction finding. U.S. Patent 5990834,12 pp.

Capet, X., J. C. McWilliams, M. J. Molemaker, and A. F. Shchepetkin, 2008: Mesoscale to submesoscale transition in the California Current System. Part I: Flow structure, eddy flux, and observational tests. J. Phys. Oceanogr., 38, 29-43, https://doi.org/10.1175/2007JPO3671.1.

Chavanne, C., P. Flament, and K.-W. Gurgel, 2010a: Interactions between a submesoscale anticyclonic vortex and a front. J. Phys. Oceanogr., 40, 1802-1818, https://doi.org/10.1175/2010JPO4055.1.

—,- D. S. Luther, and K.-W. Gurgel, 2010b: Observations of vortex Rossby waves associated with a mesoscale cyclone. J. Phys. Oceanogr., 40, 2333-2340, https://doi.org/10.1175/ 2010JPO4495.1.

Choi, Y. H., 1999: Alternating projection for maximum-likelihood source localization using eigendecomposition. IEEE Signal Process. Lett., 6, 73-75, https://doi.org/10.1109/97.752057.

Davis, R. E., 1985: Drifter observations of coastal surface currents during CODE: The method and descriptive view. J. Geophys. Res., 90, 4741-4755, https://doi.org/10.1029/JC090iC03p04741.

de Paolo, T., and E. Terrill, 2007: Skill assessment of resolving ocean surface current structure using compact-antennastyle HF radar and the MUSIC direction-finding algorithm J. Atmos. Oceanic Technol., 24, 1277-1300, https://doi.org/ 10.1175/JTECH2040.1.

— - T. Cook, and E. Terrill, 2007: Properties of HF radar compact antenna arrays and their effect on the MUSIC algorithm. Oceans 2007, Vancouver, BC, Canada, IEEE, https://doi.org/ 10.1109/OCEANS.2007.4449265.

Emery, B. M., 2018: Evaluation of alternative direction of arrival methods for oceanographic HF radars. IEEE J. Oceanic Eng., https://doi.org/10.1109/JOE.2019.2914537, in press.

, and L. Washburn, 2017: Improved direction of arrival methods for oceanographic HF radars. Conf. on Antenna Measurements and Applications, Syracuse, NY, IEEE, https:// doi.org/10.1109/CAMA.2016.7815813.
— and - 2019: Uncertainty estimates for SeaSonde HF radar ocean current observations. J. Atmos. Oceanic Technol., 36, 231-247, https://doi.org/10.1175/JTECH-D-18-0104.1.

$\longrightarrow$ - $\longrightarrow$, and J. A. Harlan, 2004: Evaluating radial current measurements from CODAR high-frequency radars with moored current meters. J. Atmos. Oceanic Technol., 21, 1259-1271, https://doi.org/10.1175/1520-0426(2004)021<1259: ERCMFC $>2.0 . \mathrm{CO} ; 2$.

Forget, P., 2015: Noise properties of HF radar measurement of ocean surface currents. Radio Sci., 50, 764-777, https://doi.org/ 10.1002/2015RS005681.

Friedlander, B., 2009: Wireless direction-finding fundamentals. Classical and Modern Direction-of-Arrival Estimation, Elsevier, 1-51, https://doi.org/10.1016/B978-0-12-374524-8.00001-5.

Gurgel, K.-W., G. Antonischki, H.-H. Essen, and T. Schlick, 1999: Wellen Radar (WERA): A new ground-wave HF radar for ocean remote sensing. Coastal Eng., 37, 219-234, https:// doi.org/10.1016/S0378-3839(99)00027-7.

Harlan, J., E. Terrill, L. Hazard, C. Keen, D. Barrick, C. Whelan, S. Howden, and J. Kohut, 2010: The Integrated Ocean Observing System high-frequency radar network: Status and local, regional, and national applications. Mar. Technol. Soc. J., 44, 122-132, https://doi.org/10.4031/MTSJ.44.6.6.

Horn, R., and C. Johnson, 1985: Matrix Analysis. Cambridge University Press, $561 \mathrm{pp}$.

Jeans, P., and R. Donnelly, 1986: Four-element CODAR beam forming. IEEE J. Oceanic Eng., 11, 296-303, https://doi.org/ 10.1109/JOE.1986.1145174.

Johnson, D. H., and D. E. Dudgeon, 1993: Array Signal Processing: Concepts and Techniques. PTR Prentice Hall, 533 pp.

Kaplan, D. M., and J. Largier, 2006: HF radar-derived origin and destination of surface waters off Bodega Bay, California. Deep-Sea Res. II, 53, 2906-2930, https://doi.org/10.1016/ j.dsr2.2006.07.012.

Kirincich, A., 2017a: HFR_DP developer package. Woods Hole Oceanographic Institution, Woods Hole, https:/github.com/ akirincich/HFR $\left\{\_\right\} D P . g i t$.

2017b: Improved detection of the first-order region for direction-finding HF radars using image processing techniques. J. Atmos. Oceanic Technol., 34, 1679-1691, https://doi.org/ 10.1175/JTECH-D-16-0162.1.

— 2019: LERA HF radar developer package. Woods Hole Oceanographic Institution, https://doi.org/10.5281/zenodo.3353662.

_ and S. J. Lentz, 2017: The importance of lateral variability on exchange across the inner shelf south of Martha's Vineyard, MA. J. Geophys. Res. Oceans, 122, 2360-2381, https://doi.org/ 10.1002/2016JC012491.

, T. de Paolo, and E. Terrill, 2012: Improving HF radar estimates of surface currents using signal quality metrics, with application to the MVCO high-resolution radar system. J. Atmos. Oceanic Technol., 29, 1377-1390, https://doi.org/ 10.1175/JTECH-D-11-00160.1.

Kohut, J. T., H. J. Roarty, and S. M. Glenn, 2006: Characterizing observed environmental variability with HF Doppler radar surface current mappers and acoustic Doppler current profilers: Environmental variability in the coastal ocean. IEEE J. Oceanic Eng., 31, 876-884, https://doi.org/10.1109/ JOE.2006.886095.

Krim, H., and M. Viberg, 1996: Two decades of array signal processing research: The parametric approach. IEEE Signal Process. Mag., 13, 67-94, https://doi.org/10.1109/79.526899.

Laws, K. E., D. M. Fernandez, and J. D. Paduan, 2000: Simulationbased evaluations of HF radar ocean current algorithms. 
IEEE J. Oceanic Eng., 25, 481-491, https://doi.org/10.1109/ 48.895355 .

- J. D. Paduan, and J. Vesecky, 2010: Estimation and assessment of errors related to antenna pattern distortion in CODAR SeaSonde high-frequency radar ocean current measurements. J. Atmos. Oceanic Technol., 27, 1029-1043, https://doi.org/10.1175/2009JTECHO658.1.

Lee, Y. U., J. Choi, I. Song, S. Member, and S. R. Lee, 1997: Distributed source modeling and direction-of-arrival estimation techniques. IEEE Trans. Signal Process., 45, 960-969, https:// doi.org/10.1109/78.564184.

Lentz, S. J., and M. R. Fewings, 2012: The wind- and wave-driven inner-shelf circulation. Annu. Rev. Mar. Sci., 4, 317-343, https://doi.org/10.1146/annurev-marine-120709-142745.

Lipa, B., B. Nyden, D. S. Ullman, and E. Terrill, 2006: SeaSonde radial velocities: Derivation and internal consistency. IEEE J. Oceanic Eng., 31, 850-861, https://doi.org/10.1109/ JOE.2006.886104.

Martinez-Pedraja, J., L. K. Shay, B. K. Haus, and C. Whelan, 2013: Interoperability of seasondes and Wellen Radars in mapping radial surface currents. J. Atmos. Oceanic Technol., 30, 2662-2675, https://doi.org/10.1175/JTECH-D-13-00022.1.

Oh, S. K., and C. K. Un, 1991: Fast initialization procedure of the alternating projection algorithm for maximum-likelihood localization of multiple sources. Signal Process., 25, 381-389, https://doi.org/10.1016/0165-1684(91)90122-Y.

Ohlmann, C., P. White, L. Washburn, E. Terrill, B. Emery, and M. Otero, 2007: Interpretation of coastal HF radar-derived surface currents with high-resolution drifter data. J. Atmos. Oceanic Technol., 24, 666-680, https://doi.org/10.1175/JTECH1998.1.

Paduan, J. D., and L. Washburn, 2013: High-frequency radar observations of ocean surface currents. Annu. Rev. Mar. Sci., 5, 115-136, https://doi.org/10.1146/annurev-marine-121211172315 .

Read, W., 1999: A maximum likelihood HF direction finding estimator for high latitude distributed signals. Defence Research Establishment Tech. Rep., 83 pp., https://apps.dtic.mil/dtic/tr/ fulltext/u2/a371117.pdf.

Romero, L., D. A. Siegel, J. C. McWilliams, Y. Uchiyama, and C. Jones, 2016: Characterizing storm water dispersion and dilution from small coastal streams. J. Geophys. Res. Oceans, 121, 3926-3943, https://doi.org/10.1002/2015JC011323.

Rypina, I. I., A. R. Kirincich, R. Limeburner, and I. A. Udovydchenkov, 2014: Eulerian and Lagrangian correspondence of high-frequency radar and surface drifter data: Effects of radar resolution and flow components. J. Atmos. Oceanic
Technol., 31, 945-966, https://doi.org/10.1175/JTECH-D-1300146.1.

Schmidt, R. O., 1986: Multiple emitter location and signal parameter estimation. IEEE Trans. Antenna Propag., 34, 276-280, https://doi.org/10.1109/TAP.1986.1143830.

Shearman, R. K., and S. J. Lentz, 2004: Observations of tidal variability on the New England shelf. J. Geophys. Res., 109, C06010, https://doi.org/10.1029/2003JC001972.

Stoica, P., and A. Nehorai, 1989: Music, maximum likelihood, and Cramer-Rao bound. IEEE Trans. Acoust. Speech Signal Process., 37, 720-741, https://doi.org/10.1109/29.17564.

__ , and K. C. Sharman, 1990: Maximum likelihood methods for direction-of-arrival estimation. IEEE Trans. Acoust. Speech Signal Process., 38, 1132-1143, https://doi.org/10.1109/ 29.57542 .

Tuncer, E., and B. Friedlander, 2009: Classical and Modern Direction-of-Arrival Estimation. Academic Press, 451 pp.

Ullman, D. S., J. O'Donnell, J. Kohut, T. Fake, and A. Allen, 2006: Trajectory prediction using HF radar surface currents: Monte Carlo simulations of prediction uncertainties. J. Geophys. Res., 111, C12005, https://doi.org/10.1029/2006JC003715.

Viberg, M., B. Ottersten, and T. Kailath, 1991: Detection and estimation in sensor arrays using weighted subspace fitting. IEEE Trans. Signal Process., 39, 2436-2449, https://doi.org/ 10.1109/78.97999.

Wang, W., and E. W. Gill, 2016: Evaluation of beamforming and direction finding for a phased array HF ocean current radar. J. Atmos. Oceanic Technol., 33, 2599-2613, https://doi.org/ 10.1175/JTECH-D-15-0181.1.

Washburn, L., E. Romero, C. Johnson, C. Gotschalk, and B. M. Emery, 2016: Antenna calibration for oceanographic radars using aerial drones. Conf. on Antenna Measurements and Applications, Syracuse, NY, IEEE, https://doi.org/10.1109/ CAMA.2016.7815751.

Wax, M., and T. Kailath, 1985: Detection of signals by information theoretic criteria. IEEE Trans. Speech Signal Process., 33, 387-392, https://doi.org/10.1109/TASSP.1985.1164557.

Wilkin, J., 2006: The summertime heat budget and circulation of southeast New England shelf waters. J. Phys. Oceanogr., 36, 1997-2011, https://doi.org/10.1175/JPO2968.1.

Wyatt, B. L. R., 2005: HF radar for real-time current, wave and wind monitoring. Hydro Int., 19, 2-3.

Ziskind, I., and M. Wax, 1988: Maximum likelihood localization of multiple sources by alternating projection. IEEE Trans. Acoust. Speech Signal Process., 36, 1553-1560, https://doi.org/ 10.1109/29.7543. 